\title{
Posttraumatische Reifung nach berufsbezogener Traumatisierung bei Einsatzkräften
}

\author{
Die vermittelnde Rolle der sozio-interpersonellen Faktoren Disclosure und Co-Rumination
}

\section{Posttraumatic Growth after Profession-Related Traumatization in Rescue Workers}

\author{
The Role of Socio-Interpersonal Factors Disclosure and Co-Rumination
}

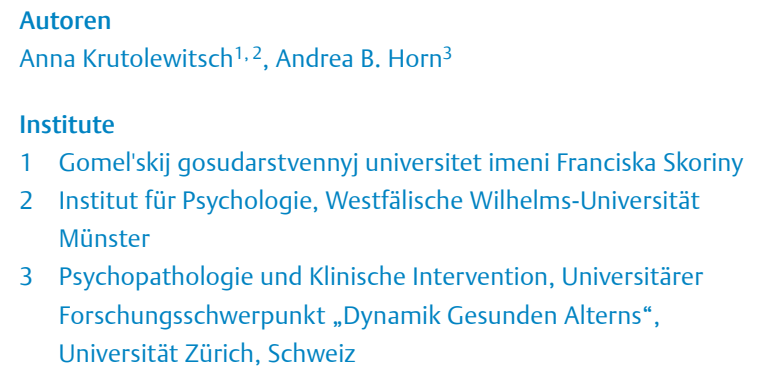

Schlüsselwörter

posttraumatische Reifung, Disclosure, Co-Rumination, sekundäre Traumatisierung, erlebte soziale Wertschätzung

\section{Keywords}

posttraumatic growth, disclosure, co-rumination, secondary traumatisation, social acknowledgement

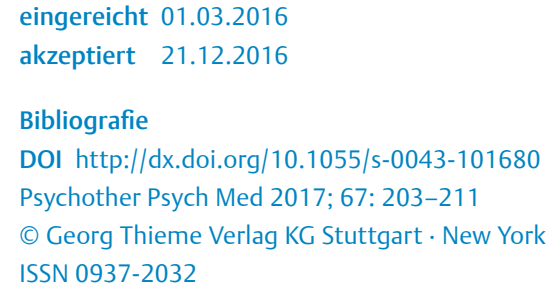

\section{ZUSAMMENFASSUNG}

Einsatzkräfte des medizinischen Rettungsdienstes und der Berufsfeuerwehr sind häufig intensiv mit Details von traumatischen Erlebnissen anderer konfrontiert. Dies kann zur Entwicklung einer sekundären Traumatisierung führen, die mit einer der primären Traumatisierung ähnelnden Symptomatik einhergeht. Andererseits sehen einige der Einsatzkräfte in ihrem Routinedienst auch eine Quelle für eine posttraumatische persönliche Reifung. Sozio-interpersonelle Prozesse, die unterschiedliche Facetten des Disclosures negativer Erfahrungen vor anderen abbilden, könnten hierbei möglicherweise eine vermittelnde Rolle spielen. Das Ziel dieser Studie ist es, den Zusammenhang zwischen Symptomen einer sekundären Posttraumatischen Belastungsstörung (PTBS) und posttraumatischen Reifung und die vermittelnde Rolle sozio-interpersoneller Variablen in einer Stichprobe von weißrussischen Einsatzkräften zu untersuchen. In einer Querschnittserhebung wurden Fragebögen zur posttraumatischen Reifung und sekundären Traumatisierung von N = 168 Einsatzkräften beantwortet. Posttraumatische Reifung war positiv mit dem von den Einsatzkräften berichteten Ausmaß an sekundärer PTBS-Symptomatik assoziiert. Zugleich zeigten dysfunktionale Muster im Zusammenhang mit traumabezogenen Disclosure, und die generelle Neigung, sich repetitiv über negative Inhalte mit Kollegen auszutauschen (Co-Rumination), positive Zusammenhänge mit Posttraumatischer Reifung. Mediatoranalysen weisen auf eine vermittelnde Funktion dieser sozio-interpersonellen Prozesse für den Zusammenhang zwischen Traumatisierungssymptomen und Reifung hin. Insgesamt sprechen die Befunde für eine zentrale Rolle sozio-interpersoneller Prozesse bei der Verarbeitung von traumatischen Ereignissen von Einsatzkräften. Dies mag ein Hinweis sein, dass sekundäre PTBS-Symptomatik zu sozialen Austauschsprozessen führen kann, die förderlich für posttraumatischer Reifung sind.

\section{ABSTRACT}

This study aims to explore the association between self-reported secondary traumatization and personal growth in a sample of Belarusians rescue workers, focusing on the mediating role of 2 socio-interpersonal processes (disclosure and co-rumination). In a cross-sectional survey, self-reported posttraumatic growth and secondary traumatization were assessed in a sample of 168 rescue workers. Posttraumatic growth was positively linked to the extent of secondary traumatization, likewise disclosure and co-rumination were positively linked to posttraumatic growth. Mediation analyses revealed that the association between secondary traumatization and posttraumatic personal growth was mediated by disclosure and co-rumination. The results thus are in favor of a socio-interpersonal perspective on posttraumatic adjustment.

\section{Einleitung}

Die Arbeit von Einsatzkräften des medizinischen Rettungsdienstes und der Berufsfeuerwehr ist nicht nur mit dem Erleben von Situa- tionen mit schwer verunglückten oder sterbenden Opfern, sondern auch mit emotional bewegendem Kontakt mit Betroffenen und Angehörigen verbunden. Derartige Erfahrungen können zur Entwick- 
lung einer sekundären Traumatisierung führen. Eine sekundäre Traumatisierung ist dadurch gekennzeichnet, dass sie ohne direkte sensorische Eindrücke des Ausgangstraumas durch wiederholte oder extreme Konfrontation mit aversiven Details des traumatischen Ereignisses, und häufig in zeitlicher Distanz zum Ausgangstrauma, entsteht. Die Symptomatik zeichnet sich durch Vermeidungsverhalten, Intrusionen und ein erhöhtes Erregungsniveau aus und überlappt stark mit klassischen Posttraumatischen Symptomen [1]. Dieser Überlappung wird in der Traumadefinition des DSM-5 Rechnung getragen, in welchem mittlerweile auch das indirekte wiederholte oder extreme Ausgesetztsein zu Details eines traumatischen Ereignisses unter das Traumakriterium fällt [2]. Da nahezu alle Angehörigen der Berufsfeuerwehr und des medizinischen Rettungsdienstes unmittelbar nach traumatischen Ereignissen mit den primären Opfern in Kontakt kommen, oder mit aversiven Details traumatischer Ereignisse anderer konfrontiert werden, gelten sie als Hochrisikopopulation für eine sekundäre Traumatisierung $[3,4]$.

Andererseits sehen einige der Einsatzkräfte in ihrem Routinedienst auch eine Quelle für positive persönliche Veränderungen. Von neuen positiven Selbsterkenntnissen bei Einsatzkräften nach berufsbezogenen Stresserlebnissen berichteten bspw. Ortlepp und Friedmann [5]. Linley und Joseph [6] zeigen in einer Metaanalyse, die 39 empirischen Studien umfasst, einen stabilen Befund hinsichtlich positiver Veränderungen nach verschiedenen Stresssituationen. Das hinter solchen Veränderungen stehende Konzept wird als „posttraumatisches Wachstum“ (engl. posttraumatic growth) bzw. „posttraumatische Reifung“ [7, 8] bezeichnet. Posttraumatische Reifung umfasst das subjektive Erleben von Veränderungen in 5 Bereichen: Intensivierung der Wertschätzung des Lebens, Entdeckung neuer Möglichkeiten, Intensivierung des Bewusstwerdens der eigenen Stärke, stärkeres Glauben und Intensivierung von persönlichen Beziehungen [7]. Diese Reifungsprozesse setzen per definitionem das Erleben stressbezogener oder traumatischer Ereignisse voraus, welche die Grundüberzeugungen einer Person erschüttern [7-9]. Wie sich die Richtung und die Stärke des Zusammenhangs zwischen PTBS-Symptomatik und anschließender posttraumatischer Reifung abbilden lässt, ist jedoch nicht gänzlich geklärt [8]. Konzeptionell sind die beiden Konstrukte klar zu unterscheiden. Empirisch konnten immer wieder positive lineare Zusammenhänge zwischen der Ausprägung von PTBS-Symptomatik nach Rettungs-Einsätzen [9], Tsunami - Flut [10], Verkehrsunfällen [11] oder Traumata im Rahmen des Holocaust [12] und einer anschließenden posttraumatischen Reifung gefunden werden. Darüber hinaus zeigt eine Meta-Analyse [13], die 87 Querschnittstudien einschloss, dass mit erhöhten PTBS-Symptomen, wie Intrusionen und Vermeidungsverhalten, auch höhere Werte an posttraumatischer Reifung einhergehen; gleichzeitig zeigte sich posttraumatisches Wachstum als negativ assoziiert mit depressiven Symptomen, während Angst, globaler Disstress und körperliche Gesundheit keine Zusammenhänge aufwiesen. Auch längsschnittlich konnten positive Korrelationen zwischen der PTBS Symptomatik und der Ausprägung posttraumatischer Reifung nachgewiesen werden [14]. Hierbei zeigen sich jedoch neben linearen als auch kurviliniare Zusammenhänge zwischen posttraumatischer Symptomatik und Reifung. Die kurviliniaren Zusammenhänge im Sinne einer U-Kurve legen nahe, dass bei mittlerer post- traumatischer Symptomatik am ehesten posttraumatische Reifung auftritt, und wurden auch in anderen Studien berichtet $[15,16]$.

Die Frage stellt sich nun, wie es nach einer Traumatisierung zu Reifung kommt, welche vermittelnden Prozesse sind hierbei nötig? Nach Tedeshi and Calhoun [7] werden 2 kognitiv-emotionale Informationsverarbeitungsprozesse als vermittelnd zwischen Traumatisierung und Reifung postuliert: Rumination auf intrapsychischer Ebene und interpersonelles Self-Disclosure. Beiden Prozessen werden, insbesondere nach erschütternden Lebensereignissen, eine emotionsregulatorische Funktion zugeschrieben. Zoellner und Maercker heben die Rolle von häufigem automatischen Ruminieren, insbesondere kurz nach einem belastenden Erlebnis, hervor, das langfristig helfen kann, schwerwiegende Ereignisse zu verarbeiten, weil es reflexive introspektive Prozesse einleitet [8]. Die theoretischen Vorannahmen konnten bestätigt werden: Absichtlich eingeleitetes ruminatives Denken weist die erwarteten positiven Zusammenhänge mit posttraumatischer Reifung auf [17-19] genauso wie Disclosure [17-20].

\section{Sozio-interpersonelle Faktoren und posttraumatische Reifung}

Die Rolle von Disclosure als Pfad zur Reifung passt zu Befunden, die der sozialen Unterstützung eine zentrale Rolle bei der posttraumatischen Reifung zuweisen [21-23]. Interessant ist hierbei, dass in Längsschnittstudien dokumentiert werden konnte, dass die Stärke des Zusammenhangs über die Zeit hinweg abnimmt; soziale Unterstützung scheint demnach am wichtigsten in zeitlicher Nähe zum Trauma zu sein [24-27]. Dabei wird betont, dass erlebte soziale Unterstützung, deren protektive Wirkung für die posttraumatische Symptomatik metaanalytisch untersucht und bestätigt wurde [28], den Prozess der posttraumatischen Reifung auch indirekt beeinflusst, indem sie sich auf das ruminative Denken auswirkt, was wiederum eine neue Bewertung eines Traumas ermöglicht und zum posttraumatischen persönlichen Wachstum führt [7].

Die Vermutung, dass es ein Wechselspiel zwischen sozialen Prozessen und ruminativer Qualität der Verhaftung mit negativen Themen gibt, wird auch im neu eingeführten Konstrukt der Co-Rumination aufgegriffen. Unter Co-Rumination wird, insbesondere im Kontext der Erforschung von Risikofaktoren für Depressionen, eine extensive und wiederkehrende Besprechung von Problemen mit nahen Freunden verstanden [29]. Im Vergleich zu dem von Maercker und Kollegen untersuchten Phänomen der dysfunktionalen Muster von Disclosure, das sowohl das Erzählverhalten über das Trauma als auch psychische und körperliche Reaktionen beim Erzählen des Traumas erfasst [30], steht bei der Co-Rumination der repetitive Charakter der negativen Dialoginhalte über die Ursachen von negativen Gefühlen im Allgemeinen und Spekulationen über deren negativen Folgen im Vordergrund. Das sich kreisende Wiederholen der Thematik ist ähnlich wie beim intrapsychischen Ruminieren, es findet allerdings im Dialog mit anderen statt. Co-Rumination wurde vorwiegend im Jugendalter untersucht und hat sich insbesondere bei weiblichen Jugendlichen als Risikofaktor für die Entstehung von Depressionen erwiesen [31-34]. Gleichzeitig ist CoRumination jedoch auf interpersoneller Ebene mit positiven Folgen verbunden: Es zeigen sich Zusammenhänge mit besseren interpersonellen Beziehungen und Freundschaften [31]. Dies ist insofern plausibel, als Selbstöffnung als Ausgangspunkt für den Aufbau psy- 
chischer Nähe gilt [35]. Wenn man entsprechend dysfunktionale Muster von Disclosure erhebt, zeigen sich erwartungsgemäß Zusammenhänge mit Symptomatik: Maercker und Kollegen konnten zeigen, dass problematische Muster von Disclosure hohe Zusammenhänge mit posttraumatischer Symptomatik aufweisen [36, 37].

Die dargestellten Befunde zu sozio-interpersonellen Prozessen und posttraumatischer Reifung passen zu der Entwicklung, dass generell in der Literatur zu Traumafolgen verstärkt auf die zentrale Rolle sozialer Ressourcen für die Traumabewältigung hingewiesen wird $[19,20,38,39]$. Soziale Nähe und Geborgenheit sind menschliche Grundbedürfnisse, deren Erfüllung als Puffer zwischen einem stresserzeugenden Ereignis und psychischer Gesundheit gesehen werden kann [40]. So schlagen Maercker und Horn [39] eine sozio-interpersonelle Perspektive auf Traumafolgephänomene vor, welche die Wichtigkeit der sozialen Wirklichkeit des Betroffenen bei der Bewältigung von Traumata unterstreicht. Interpersonelle Prozesse, die das Mitteilen von negativen Inhalten einschließen, können so also gleichzeitig innerhalb der Person problematisch sein und dennoch protektiv: Durch die Selbstöffnung wird im positiven Falle die Entstehung psychischer Nähe gefördert, wodurch sich die Qualität der engen interpersonellen Beziehungen verbessern kann. Studien zur gesellschaftlich wahrgenommenen Wertschätzung von Traumaüberlebenden illustrieren diese Annahme [39,41]: Es erweist sich als protektiv, wenn Betroffene ihre nähere und ferne soziale Umgebung als wertschätzend bzgl. ihrer Traumatisierung erfahren.

Zusammenfassend kann festgestellt werden: In den Erklärungsmodellen der Entstehung von posttraumatischer Reifung werden jenen Prozessen eine vermittelnde Rolle zugesprochen, die etwas mit ruminativen Selbstfokus und dem Mitteilen von traumatischen und negativen Inhalten an andere zu tun haben. Dabei wird von Wechselspielen zwischen intra- und interpersonellen Prozessen ausgegangen. Diese vermittelnde Rolle wurde allerdings nach unserem Wissen noch nicht explizit untersucht. Aus den Befunden in der Literatur und dem sozio-interpersonellen Modell von Maercker und Horn [39] ergibt sich die Annahme, dass die Muster von Disclosure von traumatischen Inhalten eine vermittelnde Rolle zwischen dem Erleben von und der Belastung durch die indirekte traumatische Erfahrung spielen könnten. Inhaltlich liegt weiter nahe, dass der negative Fokus mit repetitivem Charakter im Dialog mit anderen einen interpersonellen Prozess darstellt, der neben gefährdenden Aspekten potenziell protektive soziale Ressourcen mobilisiert, die wiederum ein posttraumatisches Wachstum fördern.

Die vorliegende Studie untersucht das Ausmaß der sekundären PTBS-Symptomatik in einer Stichprobe von Einsatzkräften, die berufsbedingt den traumatisierenden Ereignissen anderer ausgesetzt sind. Dabei wird erwartet, dass die berichteten Symptome sekundärer Traumatisierung einen Zusammenhang mit posttraumatischer Reifung aufweisen.

Wie eingeführt, wird dabei eine vermittelnde Rolle von Disclosure und Co-Rumination zwischen sekundärer Traumatisierung und posttraumatischem Wachstum erwartet. Die Chance zum posttraumatischen Wachstum im Zusammenhang mit einer Traumatisierung sollte insbesondere dann bestehen, wenn günstige interpersonelle Prozesse vorhanden sind, die zu verbesserter Beziehungsqualität bei nahen Beziehungen führen können. Das erwartete Mediationsmodell soll gemäß den Vorschlägen von Baron und Kenny analysiert werden [42]. Um die Reaktionen des sozialen Kontexts ebenfalls zu berücksichtigen, wird auch die wahrgenommene soziale Wertschätzung, die besser als ein konventionelles Maß an sozialer Unterstützung die PTBS-Symptomatik vorhersagt [41], hinsichtlich ihres zu erwartenden positiven Zusammenhangs mit posttraumatischer Reifung untersucht.

\section{Methoden}

\section{Stichprobe}

Die Untersuchung fand 2011 an mehreren Feuerwehrstationen und einer zentralen medizinischen Notdienststation in Stadt Gomel statt, in der die Einsatzkräfte ihre Arbeit im Schichtdienst ausüben. Die Studienteilner wurden im beruflichen Kontext angesprochen und beantworteten einzeln oder in Gruppen von ca. 10 Teilnehmenden die Fragebögen. Die informierte Einwilligung zur Studienteilnahme erfolgte nach einer kurzen Einführung durch die Studienleiterin. Die Studienteilnahme war freiwillig und anonym. An der Untersuchung beteiligten sich 168 Einsatzkräfte, darunter 53 die Mitarbeitende des medizinischen Rettungsdienstes und 115 Mitarbeitende der Berufsfeuerwehr.

Unter den befragten Feuerwehrleuten waren die Mehrzahl, dem Berufsbild entsprechend, männlich (96,5\%), während die teilnehmenden Mitarbeiter des medizinischen Rettungsdienstes mehrheitlich weiblich (66\%).

\section{Messinstrumente}

Die russischen Formulierungen der Items für die in dieser Studie angewendeten Fragebögen wurden in einem Übersetzung-Rückübersetzungsverfahren in Abstimmung mit den Originalautoren gewonnen.

Zur Erfassung der zum Zeitpunkt der Datenerhebung vorliegenden sekundären posttraumatischen Symptomatik wurde Secondary Trauma Questionnaire (STQ) verwendet. Dieser Fragebogen ist ein standardisiertes Instrument zur Selbstbeurteilung posttraumatischer Belastungssymptomatik auf sekundäres Traumaerleben [43]. Der Fragebogen enthält 18 Items auf einer 5-stufigen Likert-Skala, welche die Hauptsymptomkriterien von Posttraumatischer Belastung [2], wie kognitives Wiedererleben der sekundär erfahrenen Trauma-Situation, negative Emotionen, die mit der erlebten Situation verbunden sind, Vermeiden von Gedanken, Gefühlen, Gesprächen, die mit dem auch indirekt erlebten Trauma in Verbindung stehen, aber auch Symptome erhöhten Arousals, z. B. Konzentrationsschwierigkeiten, Einschlafschwierigkeiten, abdecken. Im Vergleich zu gängigen Selbstbeurteilungsverfahren primärer posttraumatischer Symptomatik wie z. B. dem IES-R [44] wird neben den im STQ erfassten Hauptsymptomkriterien von Posttraumatischen Belastungsstörung [2] zusätzlich Mitleidsmüdigkeit erfasst „Ich würde mich bedroht und verletzbar fühlen, wenn ich das erlebt hätte, was der oben genannten Person zugestoßen ist“. Der Fragebogen kann Werte von 18 bis 90 erreichen und wies im englischen Original eine gute interne Konsistenz auf [45]. Für die russische Variante des Fragebogens in dieser Stichprobe betrug Cronbach's 0,83. Auf der Grundlage des Cut-Off-Wertes von 38 kann die Prävalenz der sekundären Traumatisierung angegeben werden.

Zur Erfassung dysfunktionaler Muster des Offenlegens traumatischer Erfahrungen wurde Disclosure of Trauma Questionnaire 
(DTQ) eingesetzt. Der Fragebogen besteht aus 34 Items und umfasst 3 Subskalen: Die Subskala „Verschwiegenheit“ erfragt die Bedingungen der Verschwiegenheit bzgl. der traumatischen Erfahrungen anderen gegenüber (Beispielitem: „Ich mache mir zwar viele Gedanken über meine Erfahrungen, spreche aber kaum darüber“ [46]. Hingegen erfasst die Subskala „Mitteilungsdrang“ die Bedingungen des Redens über traumatische Erfahrungen (Beispielitem „Ich habe das Bedürfnis, sehr oft von dem Vorfall zu sprechen“). Die bei der Dislcosure von traumatischer Erfahrungen erlebten psychischen und körperlichen Reaktionen beim Erzählen des Traumas erfasst die letzte Subskala „emotionale Reaktion“ (Beispielitem „Ich fühle mich extrem angespannt, während ich von dem Vorfall erzähle“). Es können bei diesen Subskalen Werte von 0 bis höchstens 170 erreicht werden. Für diesen Fragebogen konnten gute psychometrische Kennwerte nachgewiesen werden [46]. Für die russische Variante des Fragebogens in dieser Stichprobe betrug Cronbach's 0,89.

Zur Erfassung der Ausprägung der Co-Rumination wurde von den Probanden Co-Rumination Questionaire (CRQ) ausgefüllt. Es handelt sich um eine 27-Items-Fragebogen [29], welcher die Neigung zur Co-Rumination, also zum wiederholten Beschäftigen mit negativen Inhalten allgemein im Gespräch zwischen Freunden misst. Der Fragebogen enthält folgende Aussagen wie: „Wenn wir über das Problem sprechen, das einer von uns hat, besprechen wir all diese Gründe, warum das Problem aufgetreten sein könnte“, „Wenn wir über das Problem sprechen, das einer von uns hat, besprechen wir jeden Teil des Problems immer wieder" und deckt somit folgende Problembereiche der Co-Rumination auf: Das Beharren am Diskutieren von Problemen, anstatt etwas anderes zu tun; Spekulationen über Ursachen und Folgen von Problemen; Spekulationen über die Teile des Problems, von denen man keine Ahnung hat; Fokus beim Diskutieren auf negative Gefühle, die in Zusammenhang mit dem Problem stehen. Der Fragebogen kann Werte von 27 und 135 erreichen. Der Fragebogen zeigte eine sehr gute interne Konsistenz. Für die russische Variante des Fragebogens in dieser Stichprobe betrug Cronbach's $\alpha=0,89$.

Zur Erfassung von posttraumatischer Reifung wurde Posttraumatic Growth Inventory (PTGI) verwendet. Der Fragebogen zur für verschiedene Opfergruppen erhebt das subjektiv wahrgenommene persönliche Wachstum. Der Fragebogen wurde von Tedeschi \& Calhoun [47] entwickelt. Die 21 Items des Fragebogens bilden die 5 Subskalen, welche Werte zwischen 0 und 105 erreichen können: neue Möglichkeiten, Beziehungen zu Anderen, persönliche Stärken, Wertschätzung des Lebens und religiöse Veränderungen. Für die russische Variante des Fragebogens in dieser Stichprobe betrug Cronbach's $\alpha=91$.

Zur Erfassung der von einem Individuum wahrgenommenen Anerkennung und Achtung seitens seiner Familie, Freunde, Umgebung und Autoritätspersonen als Reaktion auf die eigene Traumatisierung wurde Social Acknowledgment Questionnaire (SAQ) [48] eingesetzt. Der aus 16 Likert-skalierten Items bestehende Fragebogen umfasst 3 Subskalen „Anerkennung“, „Generelles Unverständnis“ und „Familiäres Unverständnis“ und beinhaltet folgende Aussagen: „Die Reaktionen meiner Bekannten waren hilfreich“, „In den ersten Tagen nach dem Vorfall haben mir viele ihre Hilfe angeboten“, „Die meisten Menschen können nicht verstehen, was ich durchgemacht habe“, „In meiner Familie werden meine Erfahrun- gen unterschätzt“. Der Fragebogen kann Werte von - 40 bis +40 erreichen. Für den Fragebogen konnten gute psychometrische Kennwerte nachgewiesen werden, Cronbach's $\alpha=0,79-0,86$ und Retestreliabilität $r_{t t}=0,76-0,89$ [48]. Für die russische Variante des Fragebogens in dieser Stichprobe betrug Cronbach's 0,83.

\section{Datenanalyse}

Alle Analysen erfolgten unter Verwendung von SPSS/PASW 18.0. Berufsgruppenunterschiede wurden mittels t-Test bzw. Chi²-Test analysiert. Zunächst wurden zur Beschreibung die bivariaten korrelativen Zusammenhänge mit Pearson Korrelationskoeffizienten berechnet. Dann wurden zur Testung der Varianzaufklärung der interessierenden Variablen hierarchische Regressionsanalysen durchgeführt. Dabei wurden schrittweise folgende Variablen eingeführt: Im ersten Schritt werden soziodemografische Variablen (i. e. Geschlecht, Alter, Bildungsniveau, Berufserfahrung) eingeführt, im zweiten Schritt dann die sozio-interpersonellen Variablen - erlebte gesellschaftliche Wertschätzung, Co-Rumination und Disclosure. Die Mediation wurde nach Baron und Kenny [42] mit linearen Regressionen untersucht. Um Variablen „Co-Rumination“ und „Disclosure“ als Mediator-Variablen bezeichnen zu können, müssen nach Baron und Kenny [42] 4 Bedingungen erfüllt werden. Der Prädiktor (posttraumatische Symptomatik der Einsatzkräfte) muss ein signifikanter Effekt auf die abhängige Variable (posttraumatische Reifung der Einsatzkräfte) nachgewiesen werden und dann ein signifikanter Effekt auf die Mediatoren Disclosure und Co-Rumination. Der Effekt des Prädiktors auf die abhängige Variable soll sich verringern, sobald in eine multivariate Regression eine der Mediator-Variablen aufgenommen wurde. Bei einem perfekten Mediatoreffekt wird der Zusammenhang zwischen der unabhängigen und abhängigen Variable vollständig durch die Mediatorvariable „absorbiert“. Zur Überprüfung der Signifikanz des indirekten Effekts zwischen der sekundären PTBS Symptomatik und der posttraumatischen Reifung über interpersonelle Variablen wurde ein SobelTest berechnet.

\section{Ergebnisse}

\section{Beschreibende Analysen der Stichprobe}

13,1 \% der Befragten erreichten gemäß dem Cut-Off-Wert [45] ein klinisches Niveau von sekundärer Traumatisierung. 60,7\% der Befragten waren über dem Cut-Off-Wert von 42 [49] in der Skala „Posttraumatic Growth Inventory“, der nach traumatischen Ereignissen für klinische bedeutsame positive Reifungsprozesse spricht. $91,1 \%$ von den 168 befragten Studienteilnehmern berichteten über mindestens eine potenziell traumatisierende Arbeitssituation, die dem A-Kriterium der posttraumatischen Belastungsstörung nach dem DSM-5 entsprach, welches, wie berichtet auch das Beobachten oder wiederholte Ausgesetztsein von Berichten von Traumata anderer einschließt. 16,7\% davon nannten 2 oder 3 solche Situationen.

$91 \%$ von den 168 befragten Studienteilnehmern berichteten über mindestens eine potenziell traumatisierende Arbeitssituation, die dem A-Kriterium der posttraumatischen Belastungsstörung nach dem DSM-5 entsprach. $17 \%$ davon nannten 2 oder 3 solche Situationen; in den meistgenannten Fällen (45\%) handelte sich um Schwerverletzte und Tote bei einem Brand oder Verkehrsunfällen, 
- Tab. 1 Deskriptive Statistik der Studienvariablen getrennt für die Feuerwehr und Rettungsdienst.

\begin{tabular}{|c|c|c|c|c|}
\hline & Gesamt & $\begin{array}{l}\text { Feuerwehr } \\
(n=115)\end{array}$ & $\begin{array}{l}\text { Rettungsdienst } \\
(n=53)\end{array}$ & $t / C h i^{2}$ \\
\hline \multicolumn{5}{|l|}{ Soziodemografische Variablen } \\
\hline Alter & $\begin{array}{l}M=31,15 \\
S D=9,55\end{array}$ & $\begin{array}{l}M=27,61, \\
S D=6,585\end{array}$ & $\begin{array}{l}M=38,83, \\
S D=10,488\end{array}$ & $\begin{array}{l}t=-7,166 \\
p<0,0001\end{array}$ \\
\hline Berufserfahrung (in Jahren) & $\begin{array}{l}M=9,38 \\
S D=8,32\end{array}$ & $\begin{array}{l}M=6,41, \\
S D=5,52\end{array}$ & $\begin{array}{l}M=15,85, \\
S D=9,66\end{array}$ & $\begin{array}{l}t=0,-6,632, \\
p=0,000\end{array}$ \\
\hline Bildungsniveau (in Jahren) & $\begin{array}{l}M=14,95 \\
S D=2,65\end{array}$ & $\begin{array}{l}M=14,83 \\
S D=2,63\end{array}$ & $\begin{array}{l}M=15,21, \\
S D=2,69\end{array}$ & $\begin{array}{l}t=2879,0, \\
p=0,562\end{array}$ \\
\hline \multicolumn{5}{|l|}{ Geschlecht } \\
\hline männlich & $77 \%(129)$ & $96,5 \%(111)$ & $34 \%(18)$ & $C h i^{2}=79,655$, \\
\hline weiblich & $23 \%(39)$ & $3,5 \%(4)$ & $66 \%(35)$ & $p<0,0001$ \\
\hline \multicolumn{5}{|l|}{ Untersuchungsvariablen } \\
\hline posttraumatische Reifung & $\begin{array}{l}M=46,63 \\
S D=17,51\end{array}$ & $\begin{array}{l}M=44,71, \\
S D=17,55\end{array}$ & $\begin{array}{l}M=50,77, \\
S D=16,85\end{array}$ & $\begin{array}{l}t=-2,10 \\
p=0,037\end{array}$ \\
\hline Subskala „persönliche Stärke“ & $\begin{array}{l}M=9,78 \\
S D=3,82\end{array}$ & $\begin{array}{l}M=9,26 \\
S D=3,96\end{array}$ & $\begin{array}{l}M=10,91 \\
S D=3,27\end{array}$ & $\begin{array}{l}t=-2,64 \\
p=0,009\end{array}$ \\
\hline Subskala „neue Möglichkeiten“ & $\begin{array}{l}M=10,50 \\
S D=4,62\end{array}$ & $\begin{array}{l}M=10,01, \\
S D=4,52\end{array}$ & $\begin{array}{l}M=11,57, \\
S D=4,71\end{array}$ & $\begin{array}{l}t=-2,05, \\
p=0,042\end{array}$ \\
\hline Subskala „religiöse Veränderungen“ & $\begin{array}{l}M=3,59 \\
S D=2,93\end{array}$ & $\begin{array}{l}M=3,27, \\
S D=2,90\end{array}$ & $\begin{array}{l}M=4,28, \\
S D=3,02\end{array}$ & $\begin{array}{l}t=-2,10 \\
p=0,037\end{array}$ \\
\hline Subskala „Wertschätzung des Lebens“ & $\begin{array}{l}M=8,65 \\
S D=3,56\end{array}$ & $\begin{array}{l}M=8,57 \\
S D=8,57\end{array}$ & $\begin{array}{l}M=8,85, \\
S D=8,85\end{array}$ & $\begin{array}{l}t=-0,448 \\
p=0,665\end{array}$ \\
\hline Subskala „Beziehung zu Anderen“ & $\begin{array}{l}M=14,10 \\
S D=6,41\end{array}$ & $\begin{array}{l}M=13,61, \\
S D=6,32\end{array}$ & $\begin{array}{l}M=15,17 \\
S D=6,51\end{array}$ & $\begin{array}{l}t=-1,473, \\
p=0,143\end{array}$ \\
\hline sekundäre Traumatisierung & $\begin{array}{l}M=29,95 \\
S D=7,96\end{array}$ & $\begin{array}{l}M=28,08, \\
S D=6,52,\end{array}$ & $\begin{array}{l}M=32,75 \\
S D=9,74\end{array}$ & $\begin{array}{l}t=-3,67, \\
p=0,002\end{array}$ \\
\hline erlebte soziale Wertschätzung & $\begin{array}{l}M=-0,76 \\
S D=5,39\end{array}$ & $\begin{array}{l}M=0,09 \\
S D=4,6\end{array}$ & $\begin{array}{l}M=-2,6 \\
S D=6,5\end{array}$ & $\begin{array}{l}t=3,077, \\
p=0,002\end{array}$ \\
\hline Subskala „Anerkennung“ & $\begin{array}{l}M=11,00 \\
S D=3,46\end{array}$ & $\begin{array}{l}M=10,7 \\
S D=3,5\end{array}$ & $\begin{array}{l}M=11,6 \\
S D=3,2\end{array}$ & $\begin{array}{l}t=-1,690 \\
p=0,093\end{array}$ \\
\hline Subskala „generelles Unverständnis“ & $\begin{array}{l}M=10,77 \\
S D=3,67\end{array}$ & $\begin{array}{l}M=9,8, \\
S D=3,3\end{array}$ & $\begin{array}{c}M=12,8, \\
S D=3,6\end{array}$ & $\begin{array}{l}t=-5,275 \\
p=0,000\end{array}$ \\
\hline Subskala „familiäres Unverständnis“ & $\begin{array}{l}M=0,99 \\
S D=2,11\end{array}$ & $\begin{array}{l}M=0,78 \\
S D=1,7\end{array}$ & $\begin{array}{l}M=1,45, \\
S D=2,7\end{array}$ & $\begin{array}{l}t=-1,932, \\
p=0,055\end{array}$ \\
\hline Co-Rumination & $\begin{array}{l}M=61,94 \\
S D=19,73\end{array}$ & $\begin{array}{l}M=59,85 \\
S D=19,41\end{array}$ & $\begin{array}{l}M=66,47, \\
S D=19,82\end{array}$ & $\begin{array}{l}t=-2,040 \\
p=0,043\end{array}$ \\
\hline Disclosure & $\begin{array}{l}M=75,03 \\
S D=19,97\end{array}$ & $\begin{array}{l}M=71,57, \\
S D=18,48\end{array}$ & $\begin{array}{l}M=82,55 \\
S D=21,17\end{array}$ & $\begin{aligned} t & =-3,41 \\
p & =0,001\end{aligned}$ \\
\hline Subskala „Verschwiegenheit“ & $\begin{array}{l}M=33,21 \\
S D=9,88\end{array}$ & $\begin{array}{l}M=31,25, \\
S D=8,40\end{array}$ & $\begin{array}{l}M=37,45, \\
S D=11,47\end{array}$ & $\begin{array}{l}t=-3,52, \\
p=0,001\end{array}$ \\
\hline Subskala „Mitteilungsdrang“ & $\begin{array}{l}M=20,95 \\
S D=6,80\end{array}$ & $\begin{array}{l}M=20,83 \\
S D=6,9\end{array}$ & $\begin{array}{l}M=21,21, \\
S D=6,6\end{array}$ & $\begin{array}{l}t=-0,33 \\
p=0,000\end{array}$ \\
\hline Subskala „Emotionale Reaktionen“ & $\begin{array}{l}M=20,87 \\
S D=7,93\end{array}$ & $\begin{array}{l}M=19,48, \\
S D=7,33\end{array}$ & $\begin{array}{l}M=23,89, \\
S D=8,39\end{array}$ & $\begin{array}{l}t=-3,28 \\
p=0,001\end{array}$ \\
\hline
\end{tabular}

in $19 \%$ der Fälle handelte sich um schwerverletzte und tote Kinder. Die anderen Fälle betrafen Verletzungen der Kollegen während des Dienstes (10\%), das Sterben von Patienten während der Hilfeleistung ( $8 \%$ ), Suizide und Suizidversuche $(4 \%)$ und andere Situationen. Dabei ist es unklar, ob die befragten Einsatzkräfte, die keine von berufsbedingten traumatischen Situationen angaben, noch keine von solchen traumatischen Situationen erlebt haben oder ob sie sich mit traumatischen Erfahrungen nicht erneut auseinandersetzen wollten und entsprechend nicht antworteten. Die soziodemografischen Angaben der Stichprobe, Mittelwerte, Standardabweichungen und die Mittelwertunterschiede der studienrelevanten Variablen sind in $>$ Tab. 1 aufgelistet. 
- Tab. 2 Korrelationen der posttraumatischen Reifung und der Prädiktorvariablen.

\begin{tabular}{|c|c|c|c|c|c|c|c|c|c|c|}
\hline & PR & D-V & D-M & D-ER & D-GW & EW-A & EW-GU & EW-FU & EW-GW & CR \\
\hline D-V & $0,56^{* *}$ & & & & & & & & & \\
\hline D-M & $0,37^{* *}$ & $0,24 * *$ & & & & & & & & \\
\hline D-ER & $0,54 * *$ & $0,65 * *$ & $0,54^{* *}$ & & & & & & & \\
\hline D-GW & $0,62 * *$ & $0,84^{* *}$ & $0,67 * *$ & $0,89 * *$ & & & & & & \\
\hline EW-A & $0,32 * *$ & $0,26^{* *}$ & $0,36 * *$ & $0,39 * *$ & $0,42 * *$ & & & & & \\
\hline EW-GU & $0,37^{* *}$ & $0,52 * *$ & $0,32 * *$ & $0,53^{* *}$ & $0,57^{* *}$ & $0,28 * *$ & & & & \\
\hline EW-FU & 0,03 & $0,16^{*}$ & 0,03 & $0,24 * *$ & $0,15^{* *}$ & $-0,14$ & $0,27 * *$ & & & \\
\hline EW-GW & $-0,06$ & $-0,25 * *$ & 0,00 & $-0,21 * *$ & $-0,20 * *$ & $0,51^{* *}$ & $-0,61 * *$ & $-0,67^{* *}$ & & \\
\hline CR & $0,39 * *$ & $0,40^{* *}$ & $0,40 * *$ & $0,51 * *$ & $0,54^{* *}$ & $0,47^{* *}$ & $0,33 * *$ & $-0,02$ & 0,08 & \\
\hline ST & $0,42 * *$ & $0,55^{* *}$ & $0,25 * *$ & $0,64^{* *}$ & $0,61 * *$ & $0,23 * *$ & $0,43^{* *}$ & $0,28 * *$ & $-0,20 * *$ & $0,44 * *$ \\
\hline
\end{tabular}

PR: Posttraumatische Reifung (PTGI - Posttraumatic Growth Inventory);D-GW: Disclosure, Gesamtwert (DTQ - Disclosure of Trauma Questionnaire);D-V: Disclosure, Subskala „Verschwiegenheit“ (DTQ );D-M: Disclosure, Subskala „Mitteilungsdrang“ (DTQ);D-ER: Disclosure, Subskala „Emotionale Reaktion“ (DTQ);EW-GW: erlebte Wertschätzung, Gesamtwert (SAQ - Social Acknowledgement Questionnaire);EW-A: erlebte Wertschätzung, Subskala „Anerkennung“ (SAQ);EW-GU: erlebte Wertschätzung, Subskala „generelles Unverständnis“ (SAQ);EW-FU: erlebte Wertschätzung, Subskala „familiäres Unverständnis“ (SAQ);CR: Co- Rumination (CRQ - Co-Rumination Questionnaire);ST: Sekundäre Traumatisierung (STQ - Secondary Trauma Questionnaire)

Mediationsanalyse: Testung der vermittelnden Rolle von sozio-interpersonellen Variablen

Zusammenhang: Symptome sekundärer Traumatisierung mit posttraumatischer Reifung Nach Baron und Kenny [42] ist der erste Schritt der Mediationsanalyse die Testung des Zusammenhangs zwischen Prädiktor und abhängiger Variable. Hier zeigte erwartungsgemäß der STQ Gesamtscore einen bedeutsamen positiven Zusammenhang mit posttraumatischer Reifung $(r=0,42$, bei $\mathrm{p}<0,001)(\triangleright$ Tab. 2).

Zusammenhang: Sozio-interpersonelle Variablen mit posttraumatischer Reifung In $>$ Tab. 3 sind die Ergebnisse der Regressionsanalyse zur Vorhersage der posttraumatischen Reifung durch sozio-interpersonelle Variablen abgetragen, die den zweiten Schritt der Mediationsanalyse darstellen und überprüfen, ob die erwarteten Mediatoren den erwarteten Zusammenhang mit der abhängigen Variable aufweisen. In diesem Fall wurden zur Vermeidung multipler Testungen und im Sinne eines konservativen Vorgehens alle interessierenden Variablen in ein Modell einbezogen. Da es keine theoretische Begründung für eine Reihenfolge gibt, wurden alle Variablen gleichzeitig eingeschlossen. Alle in die Regressionsanalyse einbezogenen Prädiktorvariablen klärten insgesamt 41 \% Varianz der posttraumatische Reifung auf.

Die Kontrollvariablen trugen $7 \%$ zur Varianzaufklärung der posttraumatischen Reifung bei. Alter und Berufserfahrung leisteten keinen signifikanten Erklärungsbeitrag in diesem Modell. Geschlecht erwies sich als die einzige der in die Analyse einbezogenen soziodemografischen Variablen, die einen signifikanten Beitrag zu dem Ausmaß posttraumatischer Reifung leistete. In diesem konservativen Modell wird posttraumatische Reifung signifikant durch Disclosure vorhergesagt; unter den Subskalen wies „Verschwiegenheit" den größten Prädiktionswert auf.

\section{Testung der Mediation des Zusammenhangs sekundäre Traumatisierung - posttraumatische Reifung durch sozio-interpersonelle Variablen}

Nun folgte der letzte Schritt einer Mediationsanalyse nach Baron und Kenny [42]. Die vermittelnde Rolle von Disclosure und Co-Rumination im Zusammenhang zwischen der sekundären PTBS Symptomatik und posttraumatischer Reifung wurde getestet, indem Symptome sekundärer Traumatisierung gleichzeitig mit Disclosure respektive Co-Rumination vorhergesagt wurde. In der $>$ Tab. 4 sind die Ergebnisse der durchgeführten Regressionsanalysen dargestellt.

Das Beta-Gewicht des Zusammenhangs zwischen sekundärer Traumatisierung und posttraumatischer Reifung verringerte sich bei Einführung der Werte des Disclosure sowie der Co-Rumination in den Regressionsmodellen. Dies kann als Hinweis auf einen zumindest partiellen Mediatoreffekt der Co-Rumination und eine vollständige Mediation durch Disclosure gewertet werden. Der SobelZ-Test ergab einen signifikanten indirekten Effekt der sekundären PTBS Symptomatik über Co-Rumination auf die posttraumatische Reifung, $z=2,89, p=0,003$. Der indirekte Effekt betrug 0,241 . Auch der indirekte Effekt der sekundären PTBS Symptomatik über Disclosure auf die posttraumatische Reifung ist signifikant, $(z=5,94$, $p<0,0001$ ) und mit 0,772 erwartungsgemäß größer.

\section{Diskussion}

In der vorliegenden Studie wurde in einer weißrussischen Population von Einsatzkräften untersucht, ob auch in dieser Population ein Zusammenhang sekundärer PTBS Symptomatik und posttraumatischer Reifung besteht und ob dieser über sozio-interpersonelle Prozesse vermittelt wird, die das Reden über negative Inhalte einschließen: Co-Rumination und traumabezogenes Disclosure. 
Erwartungsgemäß konnten lineare positive Zusammenhänge zwischen dem von den Einsatzkräften berichteten Ausmaß an sekundärer PTBS Symptomatik und posttraumatischer Reifung abgebildet werden. Diese sind vergleichbar mit in der Literatur berichteten Befunden mit anderen Stichproben [9-11, 14, 50]. An dieser Stelle ist es zu unterstreichen, dass von 168 befragten Einsatzkräf-

- Tab. 3 Vorhersage von posttraumatischer Reifung durch soziodemografische Variablen (Schritt 1) und sozio-interpersonelle Variablen (Schritt 2).

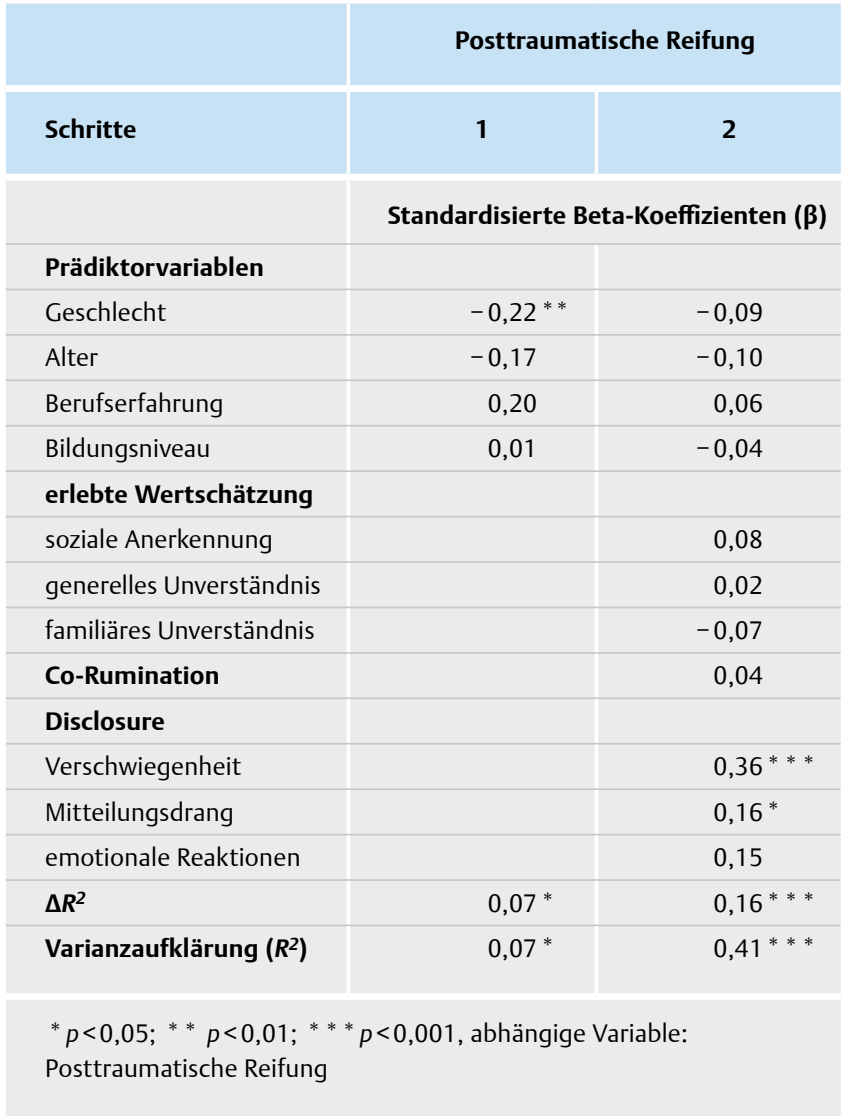

ten bei 13,1 \% ein klinisches bedeutsames Ausmaß von sekundärer Traumatisierung festgestellt werden konnte und zugleich bei $60 \%$ posttraumatische Reifung vorlag, die über dem in der Literatur vorgeschlagenen Cut-Off lag. Dieses Ergebnis stützt die Annahme, dass auch das indirekte Erleben von schwer belastenden Ereignissen ein posttraumatisches Wachstum ermöglicht [7, 8, 51]. Zudem ist festzuhalten, dass in der Stichprobe durchaus auch primärer Traumatisierung berichtet wurde. Angesichts der aktuellen Entwicklungen im Traumabereich, die immer weniger Unterschiede zwischen direkter und sekundärer Traumatisierung nahelegt, entspricht diese Überlagerung der aktuellen Sicht auf sekundäre Traumatisierung. Spezifisch für die sekundäre Traumatisierung ist die Mitleidsmüdigkeit (compassion fatigue), die in dem in dieser Studie verwendeten Instrument mit in die Ergebnisse einfließt und somit neben den phänomenologisch mit primärer Traumatisierung überlappenden Symptomen, die Spezifika von sekundärer Traumatisierung abbildet.

Die Mediationsanalysen ergaben, dass Disclosure und Co-Rumination im Zusammenhang mit sekundärer Traumatisierung zumindest eine teilweise mediierende Rolle spielen. Dies unterstützt die Annahme von Tedeshi and Calhoun [7], die schon früh das Wechselspiel zwischen intrapsychischer Rumination und interpersonellen Prozessen wie Disclosure und möglichen sozialen Ressourcen betonten. Auch die interpersonelle Perspektive des sozio-interpersonellen Modells von Maercker und Horn [39] wird dadurch unterstützt: Die gemessenen interpersonellen Prozesse, das traumabezogene Disclosure und die Öffnung von negativem Erleben gegenüber anderen im Rahmen von repetetiven Co-Ruminieren, stellen mögliche Pfade dar, über welche sekundäre Traumatisierung in Reifungsprozesse münden kann.

Betrachtet man die Ergebnisse der Unterskalen des genutzten Disclosurefragebogens, mögen die hohen Zusammenhänge der Skala „Verschwiegenheit“ mit posttraumatischer Reifung zunächst überraschen. Betrachtet man allerdings die Items dieser Skala, zeigen sich inhaltliche Überlappungen der Skala mit verstärkter innerer Auseinandersetzung („,ich denke viel darüber nach, spreche aber nicht darüber“). Möglicherweise wurde in dieser Skala auch intrapsychische Rumination erhoben, die insbesondere in zeitlicher Nähe zur Traumatisierung mit verstärkter posttraumatischer Rei-

> Tab. 4 Regressionsanalyse (schrittweise) für die Prädiktion der posttraumatischen Reifung.

\begin{tabular}{|c|c|c|c|c|c|c|}
\hline Schritte & Prädiktoren & Kriterium & $\beta$ & Total $\mathbf{R}^{2}$ & $\mathbf{T}$ & df \\
\hline 1 & Sekundäre Traumatisierung & Posttraumatische Reifung & $0,42 * * *$ & $0,184^{* * *}$ & 6,12 & 166 \\
\hline 2 & Sekundäre Traumatisierung & Co-Rumination & $0,44^{* * *}$ & $0,191^{* * *}$ & 6,27 & 166 \\
\hline 3 & Co-Rumination & Posttraumatische Reifung & $0,39 * * *$ & $0,149 * * *$ & 5,39 & 166 \\
\hline 4 & $\begin{array}{l}\text { Sekundäre Traumatisierung mit } \\
\text { Co-Rumination }\end{array}$ & Posttraumatische Reifung & $0,31 * *$ & $0,232 * * *$ & 4,21 & 165 \\
\hline 1 & Sekundäre Traumatisierung & Posttraumatische Reifung & $0,42 * * *$ & $0,184^{* * *}$ & 6,12 & 166 \\
\hline 2 & Sekundäre Traumatisierung & Diclosure & $0,61 * * *$ & $0,377 * * *$ & 9,94 & 166 \\
\hline 3 & Diclosure & Posttraumatische Reifung & $0,62 * * *$ & $0,375 * * *$ & 10,06 & 166 \\
\hline 4 & $\begin{array}{l}\text { Sekundäre Traumatisierung mit } \\
\text { Disclosure }\end{array}$ & Posttraumatische Reifung & 0,08 & $0,383 * * *$ & 1,02 & 165 \\
\hline
\end{tabular}


fung assoziiert ist. Weitere Forschung ist nötig, um die hier erstmals untersuchten Zusammenhänge besser zu verstehen. Insgesamt passen diese Ergebnisse zu Befunden [17, 38], die zeigten, dass adaptive kognitive Prozesse der Traumaverarbeitung mit posttraumatischer Reifung zusammenhängen. In den Untersuchungen $[16,52]$ erwies sich das als Risikofaktor geltende ruminative Denken als positiv assoziiert mit posttraumatischer Reifung. Auch durch eine repetitive Ursachensuche im Dialog und gemeinsame Besprechung von Konsequenzen wird ruminatives Denken gestärkt; CoRumination als auch Rumination und Disclosure korrelierten positiv miteinander [29, 32]. Hierbei ist zu vermuten, dass kognitive Prozesse, die durch Disclosure und Co-Rumination hervorgerufen wurden, mit posttraumatischer Reifung einhergehen, aber aufgrund ihrer maladaptiven Art neben posttraumatischer Reifung auch die posttraumatische Symptomatik verstärken.

Der indirekte Effekt von sekundärer PTBS Symptomatik auf posttraumatische Reifung über Disclosure war stärker als derjenige über Co-Rumination. Dies könnte darauf zurückzuführen sein, dass beim Disclosure explizit auf traumabezogenes Geschehen Bezug genommen wird. Hingegen werden bei der Co-Rumination generelle Tendenzen zum repetitiven Teilen von negativen Inhalten erfasst. Diese können, müssen jedoch nicht traumabezogene Inhalte erfassen. Weitere Forschung ist nötig, um die spezifischen Bedingungen des Mitteilens im nahen Umfeld besser zu verstehen.

Die vorliegende Studie hat eine Reihe von Einschränkungen. Unsere Daten stammen aus einer Querschnittstudie und erlauben keine kausale Interpretation der Zusammenhänge zwischen den untersuchten Variablen. Eine längsschnittliche Untersuchung könnte den zeitlichen Zusammenhang der beschriebenen Variablen klären und damit weitere Hinweise auf mögliche Kausalbeziehung geben. Eine weitere bedeutende Einschränkung ist die Gültigkeit von Selbstauskünften; so können die subjektiven Einschätzungen eigener posttraumatischer Reifung unzuverlässig wirken. Zugleich ist es bekannt, dass die Betroffenen nach einem Trauma neben erhöhten Werten an posttraumatischer Symptomatik auch von dem Zuwachs an innerer Reife und einem neu definierten Lebenssinn berichten. Die Ergebnisse dieser Studie, die Mitarbeiter des weißrussischen Feuerwehr- und Rettungsdienstes untersuchte, dürfen nur begrenzt auf andere Länder, z. B. westeuropäische und andere Traumaopfergruppen generalisiert werden. Soziokulturelle Unterschiede sind durchaus nicht auszuschließen.

Schlussfolgernd zeigte sich in der vorliegenden Arbeit, dass es Einsatzkräften, indem sie sich mit menschlichem Leid und verschiedenen Notlagen auseinandersetzen, doch gelingt, einen Zuwachs an innerer Reife und positiven persönlichen Veränderungen zu gewinnen. Das Aufdecken von traumatischen Ereignissen, insbesondere das emotionale Disclosure, bildet eine günstige Basis für weitere kognitiv-emotionale Traumaverarbeitung, die zum Wachstum in verschiedenen Lebensbereichen führt. Auch wenn viele Fragen offenbleiben, unterstreichen die Ergebnisse die zentrale Rolle von interpersonellen Prozessen im Kontext von Trauma und dessen Verarbeitung.

\section{FAZIT FÜR DIE PRAXIS}

Es hat sich herausgestellt, dass etwa zwei Drittel der Einsatzkräfte in ihrem Routinedienst auch eine Quelle für eine posttraumatische persönliche Reifung fanden. Maßgeblich für die posttraumatischen persönlichen Reifungsveränderungen sind sozio- interpersonelle Prozesse, wie das Offenlegen traumabezogener Gedanken und Gefühle (Disclosure) und der wiederholte Austausch über negative Inhalte mit Kollegen (Co-Rumination). Diese sozio-interpersonellen Prozesse sind also Ressourcen für das posttraumatische Wachstum. Gleichzeitig gilt aber auch in der Praxis zu beachten, dass dysfunktionales traumabezogenes Offenlegen und Co-Rumination, obschon sie reflexive introspektive und günstige soziale Prozesse einleiten, gleichzeitig Risikofaktoren für psychopathologische Symptomatik darstellen können.

Interessenkonflikt

Die Autoren geben an, dass kein Interessenkonflikt besteht.

Literatur

[1] Reinhard F, Maercker A. Sekundäre Traumatisierung, Posttraumatische Belastungsstörung, Burnout und Soziale Unterstützung bei medizinischem Rettungspersonal. Z Med Psychol 2004; 13: 29-36

[2] American Psychiatric Association. Diagnostic and Statistical Manual of Mental Disorders (DSM-5). Arlington: American Psychiatric Publishing; 2013

[3] Teegen F, Domnick A, Heerdegen M. Hochbelastende Erfahrungen im Berufsalltag von Polizei und Feuerwehr. VPP 1997; 4: 583-599

[4] Wagner D, Heinrichs M, Ehlert U. Prevalence of symptoms of posttraumatic stress disorder in German professional firefighters. Am J Psychiatry 1998; 12: 1727-1732

[5] Ortlepp K, Friedman M. Prevalence and correlates of secondary traumatic stress in Workplace Lay Counselors. J Trauma Stress 2002; 15: 213-222

[6] Linley PA, Joseph S. Positive change following trauma and adversity: A review. J Trauma Stress 2004; 17: 11-21

[7] Tedeschi RG, Calhoun LG. Posttraumatic growth: conceptual foundations and empirical evidence. Psychol Inq 2004; 15: 1-18

[8] Zoellner T, Maercker A. Posttraumatic growth in clinical Psychology A critical review and introduction of a two component model. Clin Psychol Rev 2006; 26: 626-653

[9] Linley PA, Joseph S. The positive and negative effects of disaster work: A preliminary investigation. J Loss Trauma 2006; 11: 229-245

[10] Tang C. 2007; Posttraumatic growth of Southeast Asian survivors with physical injuries: six months after the 2004 Southeast Asian earthquake - Tsunami. The Australian Journal of Disaster and Trauma Studies. Retrieved October 15, 2008 from http://www.massey.ac.nz/ trauma/ issues/previous.shtml\#2007-1

[11] Zoellner T, Rabe S, Karl A et al. Posttraumatic growth in accident survivors: Openness and optimism as predictors of its constructive and illusory sides. J Clin Psychol 2008; 64: 245-263 
[12] Lurie-Beck J, Liossis P, Gow K. Relationships between psychopathological and demographic variables and posttraumatic growth among holocaust survivors. Traumatology 2008; 14: 28-39

[13] Helgeson VS, Reynolds KA, Tomich PL. A meta-analytic review of benefit finding and growth. J Consult Clin Psychol 2006; 74: 797-816

[14] Lowe SR, Manove EE, Rhodes JE. Posttraumatic stress and posttraumatic growth among low-income mothers who survived hurricane Katrina. J Consult Clin Psychol 2013; 81: 877-889

[15] Solomon Z, Dekel R. Posttraumatic stress disorder and posttraumatic growth among Israeli ex-pows. J Trauma Stress 2007; 20: 303-312

[16] Kleim B, Ehlers A. Evidence for a curvilinear relationship between posttraumatic growth and posttrauma psychopathology. J Trauma Stress 2009; 22: 45-52

[17] Currier J, Lisman R, Harris I et al. Cognitive processing of trauma and attitudes toward disclosure in the first six months after military deployment. J Clin Psychol 2013; 69: 209-221

[18] Taku K, Cann A, Tedeschi R et al. Intrusive versus deliberate rumination in posttraumatic growth across US and Japanese samples. Anxiety Stress Coping 2008; 20: 1-8

[19] Taku K, Tedeschi R, Cann A et al. The culture of disclosure: Effects of perceived reactions to disclosure on posttraumatic growth and distress in Japan. J Soc Clin Psychol 2009; 29: 1226-1243

[20] Smyth JM, Hockemeyer JR, Tulloch H. Expressive writing and post-traumatic stress disorder: Effects on trauma symptoms, mood states, and cortisol reactivity. Br J Health Psychol 2008; 13: 85-93

[21] Salsman JM, Segerstrom SC, Brechting EH et al. Posttraumatic growth and PTSD symptomatology among colorectal cancer survivors: A 3 -month longitudinal examination of cognitive processing. Psychooncology 2009; 18: 30-41

[22] Dekel S, Ein-Dor T, Solomon Z. Posttraumatic growth and posttraumatic distress: A longitudinal study. Psychol Trauma 2012; 4: 94-101

[23] Levine SZ, Laufer A, Stein E et al. Examining the relationship between resilience and posttraumatic growth. J Trauma Stress 2009; 22: 282-286

[24] Park CL, Cohen LH, Murch R. Assessment and prediction of stressrelated growth. J Pers 1996; 64: 71-105

[25] Butler LD, Blasey CM, Garlan RW et al. Posttraumatic growth following the terrorist attacks of September 11, 2001: Cognitive, coping, and trauma symptom predictors in an internet convenience sample. Traumatology 2005; 11: 247-267

[26] Low C, Stanton A, Thompson $\mathrm{N}$ et al. Contextual life stress and coping strategies as predictors of adjustment to breast cancer survivorship. Ann Behav Med 2006; 32: 235-244

[27] Danhauer S, Case D, Tedeschi R et al. Predictors of posttraumatic growth in women with breast cancer. Psychooncology 2013; 22: 2676-2683

[28] Brewin CR, Andrews B, Valentine JD. Meta-analysis of risk factors for posttraumatic stress disorder in trauma-exposed adults. J Consult Clin Psychol 2000; 68: 748-766

[29] Rose AJ. Co-Rumination in the friendships of girls and boys. Child Dev 2002; 73: 1830-1843

[30] Müller ], Beauducel A, Raschka J et al. Kommunikationsverhalten nach politischer Haft in der DDR. Entwicklung eines Fragebogens zum Offenlegen der Traumaerfahrungen. ZfPP 2000; 4: 413-427

[31] Rose AJ, Carlson W, Waller EM. Prospective associations of co-rumination with friendship and emotional adjustment: Considering the socioemotional trade-offs of co-rumination. Dev Psychol 2007; 43: 1019-1031

[32] Calmes CA, Roberts JE. Rumination in interpersonal relationships: Does co-rumination explain gender differences in emotional distress and relationship satisfaction among college students? Cognit Ther Res 2008; 32: 577-590
[33] Ciesla JA, Dickson KS, Anderson NL et al. Negative repetitive thought and college drinking: angry rumination, depressive rumination, co-rumination, and worry. Cognit Ther Res 2011; 35: 142-150

[34] Curci A, Rime B. The temporal evolution of social sharing of emotions and its consequences on emotional recovery: a longitudinal study. Emotion 2012; 12: 1404-1414

[35] Reis HT, Shaver P. Intimacy as an interpersonal process. Handbook of personal relationships 1988; 24: 367-389

[36] Mueller J, Moergeli $\mathrm{H}$, Maercker A. Disclosure and social acknowledgement as predictors of recovery from posttraumatic stress: A longitudinal study in crime victims. Can J Psychiatry 2008; 53 : 160-168

[37] Pielmaier L, Maercker A. Psychological adaptation to life-threatening injury in dyads: The role of dysfunctional disclosure of trauma. Eur J Psychotraumatol 2011; 2: 1-12

[38] Phelps LF, Williams RM, Raichle KA et al. The importance of cognitive processing to adjustment in the 1 st year following amputation. Rehabil Psychol 2008; 53: 28-38

[39] Maercker A, Horn A. A socio-interpersonal perspective on PTBS: the case for environments and interpersonal processes. Clin Psychol Psychother 2013; 20: 465-481

[40] Sommer G, Fydrich T. Soziale Unterstützung, Diagnostik, Konzepte, Fragebogen F-SOZU. Tübingen: Deutsche Gesellschaft für Verhaltenstherapie; 1989

[41] Müller J, Maercker A. Disclosure und wahrgenommene gesellschaftliche Wertschätzung als Opfer als Prädiktoren von PTB bei Kriminalopfern. Z Klin Psychol Psychother (Gott) 2006; 35: 49-58

[42] Baron RM, Kenny DA. The moderator-mediator variable distinction in social psychological research: Conceptual, strategic and statistical considerations. J Pers Soc Psychol 1986; 51: 1173-1182

[43] Motta RW, Joseph JM. The secondary trauma questionnaire. unpublished scale New York: Hofstra University; 1998

[44] Weiss DS, Marmar CR. The impact of event scale - revised. In Wilson JP, Keane TM, (Hrsg.). Assessing psychological trauma and PTSD. NewYork: Guilford; 1996: 399-411

[45] Motta RW, Newman CL, Lombardo KL et al. Objective assessment of secondary trauma. Int J Emerg Ment Health 2004; 6: 67-74

[46] Müller J, Beauducel A, Raschka J et al. Kommunikationsverhalten nach politischer Haft in der DDR. Entwicklung eines Fragebogens zum Offenlegen der Traumaerfahrungen. ZfPP 2000; 4: 413-427

[47] Tedeschi RG, Calhoun LG. The posttraumatic growth inventory: measuring the positive legacy of trauma. J Trauma Stress 1996; 9: 455-471

[48] Maercker A, Müller ]. Social acknowledgement as a victim or survivor: a scale to measure a recovery factor of PTSD. J Trauma Stress 2004; 17 : 345-351

[49] Polatinsky S, Esprey Y. An assessment of gender differences in the perception of benefit resulting from the loss of a child. J Trauma Stress 2000; 13: 709-718

[50] Nishi D, Matsuoka Y, Kim Y. Posttraumatic growth, posttraumatic stress disorder and resilience of motor vehicle accident survivors. Biopsychosoc Med 2010; 4: 7

[51] Nolen-Hoeksema S, Davis C. Theoretical and methodological issues in the assessment and interpretation of posttraumatic growth. Psychol Inq 2004; 15: 60-65

[52] Calhoun L, Cann A, Tedeschi R. A correlational test of the relationship between posttraumatic growth, religion and cognitive processing. J Trauma Stress 2000; 13: 521-527 